\title{
Avaliação das propriedades físico-mecânicas de argamassa com utilização de resíduos de fundição
}

\section{(Physical-mechanical properties evaluation of mortar using foundry scrap)}

\author{
E. S. Rufino ${ }^{1}$, A. G. S. Galdino ${ }^{2}$ \\ ${ }^{1}$ Faculdade de Tecnologia Arthur de Azevedo - FATEC, R. Ariovaldo Silveira Franco 567, \\ Jardim 31 de Março, Mogi Mirim, SP 13801-005 \\ ${ }^{2}$ Instituto Federal de Ensino, Pesquisa e Extensão do Espírito Santo- IFES, Av. Vitória 1729, \\ Jucutuquara, Vitória, ES 29040-780 \\ andregsg@ifes.edu.br
}

\begin{abstract}
Resumo
O grande volume de resíduos sólidos gerados diariamente pelas indústrias vem causando grandes danos ao meio ambiente, em virtude do destino incorreto, negligência humana e pelas grandes áreas ocupadas para o descarte. O presente trabalho objetivou desenvolver um estudo para a produção de mistura de argamassa utilizada na construção civil, com reutilização de resíduos de fundição, tais como escória de fornos por indução e areia calcinada de macharia, com adição de resina em pó de Poli (cloreto de vinila) (PVC) NORVIC P72HA e um aditivo plastificante liquido (Sulfonol NSDC) na composição da argamassa onde a intenção é reduzir a absorção de água no material e melhorar suas propriedades físicas e mecânicas. Foram confeccionados 50 corpos de prova de mistura de argamassa, sendo dez de mistura de argamassa atualmente comercializada e quarenta de composições propostas, visando sua diferenciação através de ensaios físicos de absorção de água, porosidade aparente e densidade aparente e de ensaio mecânico, onde se avaliou o limite de resistência à compressão. De acordo com os resultados obtidos, pode-se constatar que as composições propostas apresentaram resultados nos ensaios físicos de $30 \%$ a menos na absorção de água e 31\% a menos na porosidade aparente. Os resultados dos ensaios mecânicos ficaram em conformidade com as especificações da NBR 8953 [1], porque os valores para a argamassa, quando comparados com os valores utilizados para concreto (com adição de brita), demonstraram propriedades semelhantes ao concreto C20 grupo I de resistência, para ser utilizado na construção civil. Porém, devido ao alto preço da resina (PVC) NORVIC P72HA, sua produção ainda não se torna atrativa. Recomenda-se, com o objetivo de viabilizar a utilização da resina, fazer um estudo para verificar qual a menor proporção de adição da mesma que seja capaz de reproduzir os resultados de propriedades físico-mecânicas recomendadas pela literatura.
\end{abstract}

Palavras-chave: resíduos sólidos industriais, concreto, reciclagem.

\begin{abstract}
The daily large volume of solid waste generated by industries has caused great harm to the environment, due to the incorrect destination, human negligence and the large areas occupied for disposal. The present work aimed to develop a study for the production of mortar mixture to be used in construction, by using foundry scrap such as slag from induction furnaces and calcined core sand, with the addition of poly (vinyl chloride) resin powder (PVC) NORVIC P72HA and a liquid plasticizer additive (Sulfonol NSDC) in the concrete mass composition which intention is to reduce water absorption of the material and improve their physical and mechanical properties. Fiftys amples have been made of concrete mass, in which ten of those samples were made of traditional concrete mass and forty of defined compositions. Samples were submitted to physical essays of water absorption (AA), apparent porosity (AP) and apparent gravity (AG) and to mechanical essays of ultimate compression strength (UCS). According to the results obtained, it can be seen that results in mortar proposed composition presented 30\% less water absorption and $31 \%$ less apparent porosity. Results of mechanical essays were in accordance with the specifications of Brazilian standard NBR 8953 [1], because even without the addition of gravel in its composition the alternative compositions showed similar properties to concrete strength of C20 group I, to be used in construction. However, due to the high cost of resin poly (vinyl chloride) (PVC) P72HA NORVIC, their production has not yet become attractive. It is recommended, in order to enable the use of resin, to do a research to see what the lowest proportion of addition of the same that is capable of reproducing the results of physical-mechanical properties recommended in the literature.
\end{abstract}

Keywords: industrial solid waste, concrete, recycling.

\section{INTRODUÇÃO}

O processo produtivo em qualquer setor industrial gera uma grande quantidade de resíduos, tanto na produção de bens duráveis como não duráveis. Todo resíduo descartado de forma descontrolada passou a ser um problema sério uma vez que ocupam grandes áreas e contaminam o meio ambiente. Há grande preocupação com essa geração de resíduos industriais, não apenas porque esse resíduo gera custo agregado ao produto, bem como pode gerar problemas com as resoluções e leis que dispõem sobre o assunto. No Brasil há a lei n . 12.305, de 02 de agosto de 2010, que institui a política nacional de 
resíduos sólidos [2]. A solução normalmente encontrada pelas empresas é o descarte dos resíduos em aterros sanitários e lixões. Porém, na estrutura das grandes cidades, fica cada vez mais difícil encontrar espaços físicos para essas obras, devido ao crescimento populacional nos centros urbanos, à dificuldade em retirar licenças ambientais e à alta valorização dos terrenos em algumas áreas.

A necessidade ambiental de reciclar resíduos industriais tem motivado e ampliado cada vez mais à pesquisa científica e a busca por novas tecnologias [3-5], com o intuito de diminuir o impacto ambiental e gerar produtos alternativos para serem usados comercialmente.

Segundo a ABNT NBR 10004 [6], que classifica os resíduos sólidos quanto aos seus riscos potenciais ao meio ambiente e à saúde pública, para que estes resíduos possam ter manuseio e destinação adequados, o resíduo de fundição em questão pode ser classificado como Classe II - A. O destino final de tal material, segundo a norma da ABNT NBR 10004 - Resíduos Sólidos Classificação [6], cuja classe é II - A - não perigoso e não inerte, se dá em aterros sanitários por não ter mais utilidade para as empresas, e por ter propriedades como biodegradabilidade, combustibilidade ou solubilidade em água. Aquilo que, no passado, era identificado pelas indústrias como um grande problema ambiental e economicamente caro, como dar um destino correto aos resíduos produzidos, está se tornando uma premissa básica para todos os setores que esperam ter seus produtos e processos otimizados.

A grande disputa por mercado não admite mais que empresas negligenciem questões como desperdício de materiais, gestão ineficiente dos processos de produção e, mais recentemente, a emissão, controle e reciclagem dos seus resíduos. A consagração do processo de reciclagem dentro da própria indústria (como por exemplo, na fabricação de ferro fundido), bem como a incorporação de resíduos como areia de fundição e escória na construção civil, transformou definitivamente a questão de gerenciamento e reaproveitamento de subprodutos muito importantes. Atualmente esta questão tem enorme importância para o desenvolvimento sustentável, que procura satisfazer as necessidades da geração atual, sem comprometer as gerações futuras. Atingindo uma condição aceitável de desenvolvimento social, econômico e de realização humana que preserve os recursos da terra, mantendo as funções e componentes do ecossistema equilibrados, para manter uma adequada condição de vida para as pessoas e outros seres vivos.

Um fator importante no incentivo da reciclagem dos resíduos industriais é evitar problemas de contaminação e degradação do meio ambiente em função da natureza dos resíduos, em especial os resíduos sólidos. Estes podem gerar danos na atmosfera, solo, lençol freático e rios, durante todo seu ciclo de vida, seja nas dependências da empresa ou no seu destino final. Resíduos de produtos químicos, subprodutos sólidos da indústria siderúrgica, resíduo do corte de pedras ornamentais, resíduos de demolições entre outros se enquadram nesta situação.

Para encontrar novos materiais alternativos para aplicação na construção civil, universidades, empresas do setor privado e associações têm trabalhado de forma insistente em pesquisas, Desta forma, novos produtos podem ser criados e suas propriedades garantidas junto as normas estabelecidas pela ABNT/CETESB, que regulamentam e controlam adequadamente a reutilização, redução e disposição final de resíduos sólidos [7]. Além disso, o material alternativo tem que apresentar um custo final reduzido para que possa competir com os materiais utilizados atualmente. Esses são alguns dos desafios encontrados no caminho de quem investe em pesquisas para se reciclar algum material. Como existe uma infinidade de possibilidades para a utilização de um resíduo, é indicado que se trabalhe seguindo alguns parâmetros já estabelecidos, buscando alternativas já estudadas por outros pesquisadores. Impedir a geração de resíduos mediante a proibição de produzir ou de consumir é, no entanto, uma alternativa quase sempre falsa, que conduz a outros tipos de problemas: pobreza, desemprego e deslocamento da produção para outras regiões [8]. O que se coloca às indústrias é a busca de novas tecnologias, a racionalização na utilização de recursos eliminando ou reduzindo perdas, fugas, o aperfeiçoamento do sistema produtivo (eco eficiência) e sua integração interna e externa para efetivar as transformações necessárias ao atual ambiente de negócios [8].

Muitos trabalhos têm sido publicados mostrando a utilização do resíduo de areia de fundição para usos gerais [9-12], onde um desses usos é para argamassas. Os finos de areia de uma fundição e sua incorporação em argamassa de cimento foram caracterizados, concluindo que a incorporação desses resíduos pode ser vantajosa por diminuir o consumo de agregado fino e cimento, e que contribui para reduzir o descarte de resíduos em aterros sanitários [13]. O resíduo de areia de moldagem (areia de retorno) e areia contida em exaustores, provenientes da mistura de areia de moldagem (areia de exaustão) foram utilizados em substituição ao agregado, onde os resultados indicaram redução na resistência mecânica quando comparados com amostras de argamassa padrão [14]. Areia descartada de fundição foi utilizada para produzir peças de concreto utilizados em pavimentos intertravados [15]. Com os resultados obtidos, foi observado que é possível utilizar resíduo de areia de fundição como agregado para fabricação de peças de concreto para pavimentação. A areia descartada de fundição foi caracterizada e constatou-se a presença de elementos potencialmente tóxicos, tais como cromo, cobre, cobalto, níquel, zinco, alumínio, ferro, manganês, considerados não perigosos pela atual normatização.

Esse trabalho teve por objetivo avaliar as propriedades físico-mecânicas de quatro composições de massa de concreto utilizando-se resíduo de fundição, a saber: porosidade aparente, densidade aparente, absorção de água e limite de resistência à compressão.

\section{MATERIAIS E MÉTODOS}

A areia calcinada de macharia foi coletada na Fundição da TRW Automotive Ltda., Planta de Engenheiro Coelho, SP, Rod. SP 332, km 162, Distrito Industrial, com um descarte médio de 590 ton por ano, conforme Tabela I. 
Tabela I - Quantidade de areia calcinada descartada por ano.

[Table I - Calcined sand quantity discarded per year.]

\begin{tabular}{cccccccc}
\hline \multirow{2}{*}{ Resíduo } & \multicolumn{7}{c}{ Quantidade por ano (ton) } \\
& 2005 & 2006 & 2007 & 2008 & 2009 & 2010 & 2011 \\
\hline Areia calcinada & 685,90 & 894,20 & 895,60 & 419,90 & 497,20 & 355,60 & 405,30 \\
Média & & & & 593,39 \\
\hline
\end{tabular}

Obs.: em 2011 não foram apontados os meses de outubro, novembro e dezembro. Fonte: TRW Automotive Ltda., planta Engenheiro Coelho, SP.

Tabela II - Quantidade de escória descartada por ano.

[Table II - Scrap quantity discarded per year.]

\begin{tabular}{cccccccc}
\hline \multirow{2}{*}{ Resíduo } & \multicolumn{7}{c}{ Quantidade por ano (ton) } \\
& 2005 & 2006 & 2007 & 2008 & 2009 & 2010 & 2011 \\
\hline $\begin{array}{c}\text { Escória de } \\
\text { forno a Indução } \\
\text { Média }\end{array}$ & $1.812,90$ & $1.939,80$ & $2.271,60$ & $2.083,60$ & $1.491,90$ & $1.872,90$ & $1.573,50$ \\
& & & & $1.863,74$ & & & \\
\hline
\end{tabular}

Obs.: no ano de 2011 não foram apontados os meses de outubro, novembro e dezembro. Fonte: TRW Automotive Ltda planta Engenheiro Coelho, SP.

Tabela III - Composição da mistura de argamassa: P (padrão); C1 (proposto 1); C2 (Proposto 2); C3 (proposto 3); C4 (proposto 4). [Table III - Mortar mixture composition: P (standard); C1 (proposition 1); C2 (proposition 2); C3 (proposition 3); C4 (proposition 4).]

\begin{tabular}{cccccc}
\hline Componente & Padrão & $\mathrm{C} 1$ & $\mathrm{C} 2$ & $\mathrm{C} 3$ & $\mathrm{C} 4$ \\
\hline Cimento & $13,51 \%$ & $10,0 \%$ & $13,51 \%$ & $13,51 \%$ & $13,51 \%$ \\
Areia & $32,43 \%$ & $16,0 \%$ & $16,00 \%$ & $32,43 \%$ & $32,43 \%$ \\
Areia calcinada de & - & $16,0 \%$ & $16,00 \%$ & - & - \\
macharia & - & - & - & - & $4,0 \%$ \\
Escória & $10,81 \%$ & $10,0 \%$ & $10,81 \%$ & $10,81 \%$ & $10,81 \%$ \\
Água & $0,2 \%$ emrelação & - & $0,2 \%$ em relação & $0,2 \%$ em relação & $0,2 \%$ em relação \\
Aditivo Plastificante & $\mathrm{a} \%$ cimento & $-\mathrm{a} \%$ cimento & $\%$ cimento & $\mathrm{a} \%$ cimento \\
Resina (PVC) NORVIC & - & $-4,0 \%$ & - & $4,0 \%$ & - \\
P72HA & - & $0,3 \%$ em relação & - & - & - \\
Aditivo sulfonol NSDC & - & $\mathrm{a} \%$ cimento & - & & \\
\hline
\end{tabular}

A escória de forno a indução utilizada no trabalho foi coletada na mesma fundição, com um descarte médio de 1.860 ton por ano, Tabela II. A escória foi triturada em moinho de bolas por 40 min e peneirada em peneira ABNT 80.

O NORVIC P72HA foi fornecido pela Braskem S/A (S. Paulo, SP), enquanto o aditivo sulfonol NSDC foi fornecido pela QGP Química (Laranjal Paulista, SP).

As amostras foram divididas em cinco composições de mistura de argamassa: P (padrão - cimento, areia, água, aditivo plastificante); $\mathrm{C} 1$ (padrão mais escória, areia calcinada de macharia, resina de (PVC) NORVIC P72HA e aditivo Sulfonol NSDC); C2 (Padrão mais areia calcinada de macharia), C3 (Padrão mais Resina de (PVC) NORVIC P72HA) e C4 (Padrão mais Escória de forno a indução). As quantidades percentuais das composições $\mathrm{P}, \mathrm{C} 1, \mathrm{C} 2, \mathrm{C} 3, \mathrm{C} 4$ e C5 estão na Tabela III.
Foram confeccionados 20 corpos de prova por composição, com dimensões de $12,1 \mathrm{~mm}$ de diâmetro por $35 \mathrm{~mm}$ de altura, utilizando carga de prensagem igual a 2500 kgf. Os corpos de provas foram confeccionados através de uma adaptação das exigências da NBR 5738 [17] e NBR 5739 [18], que citam que os corpos de prova devem ter altura igual ao dobro do diâmetro. Entretanto, não há, nessas normas, moldes com as dimensões utilizadas no trabalho e nem é citada a questão de prensagem. A cura dos corpos de prova foi feita ao ar, e não em ambiente controlado de umidade relativa ou submerso em água saturada de cal. O tempo de cura utilizado para cada composição foi de $7 \mathrm{~d}$ e $28 \mathrm{~d}$.

Os corpos de prova foram submetidos a ensaios físicos de absorção de água, densidade aparente e porosidade aparente, bem como ensaio mecânico de resistência à compressão. Os resultados dos ensaios físicos foram obtidos através da 
média aritmética de cinco corpos de prova e calculados através das equações (A), (B) e (C) respectivamente.

$$
\begin{aligned}
& \mathrm{AA} \%=\left(\frac{\mathrm{P}_{U}-\mathrm{P}_{\mathrm{S}}}{\mathrm{P}_{\mathrm{S}}}\right) \cdot 100 \\
& \operatorname{DA}\left(\mathrm{g} / \mathrm{cm}^{3}\right)=\left(\frac{\mathrm{P}_{\mathrm{S}}}{\mathrm{P}_{\mathrm{S}}-\mathrm{P}_{\mathrm{I}}}\right) \cdot 100 \\
& \operatorname{PA}(\%)=\left(\frac{\mathrm{P}_{U}-\mathrm{P}_{S}}{\mathrm{P}_{U}-\mathrm{P}_{\mathrm{I}}}\right) \cdot 100
\end{aligned}
$$

nas quais $\mathrm{P}_{\mathrm{I}}$ é o peso imerso, $\mathrm{P}_{\mathrm{S}}$ é o peso seco e $\mathrm{P}_{\mathrm{U}}$ é o peso úmido [19].

O ensaio de compressão foi feito em máquina Emic DL2000, célula Trd 27, extensômetro Trd 1, programa Tesc 3.04 e taxa de carregamento $0,30 \mathrm{MPa} / \mathrm{s}$. Os resultados foram obtidos pela média aritmética de cinco corpos de prova e calculados por meio das equações (D) e (E), respectivamente [18].

$$
\begin{gathered}
\mathrm{S}\left(\mathrm{mm}^{2}\right)=\left(\frac{\pi \cdot \mathrm{D}^{2}}{4}\right) \\
\mathrm{T}(\mathrm{MPa})=\left(\frac{\mathrm{F}}{\mathrm{S}}\right)
\end{gathered}
$$

nas quais D é o diâmetro do corpo de prova, F é acarga de compressão, S é a área da seção transversal do corpo de prova e T é a tensão de compressão.

\section{RESULTADOS E DISCUSSÃO}

A Tabela IV apresenta os resultados dos ensaios físicos de absorção de água (AA), porosidade aparente (PA) e densidade aparente (DA), dos corpos de prova do padrão, do proposto 1 , proposto 2 , proposto 3 e proposto 4 com tempo de cura de $7 \mathrm{~d}$.

Os corpos de prova do proposto 2 não apresentaram integridade física após ficarem imersos em água para a realização dos ensaios físicos, e por esse motivo não

Tabela IV - Resultados dos ensaios físicos de $7 \mathrm{~d}$. [Table IV - Results for 7 days physical essays.]

\begin{tabular}{cccc}
\hline Material & AA $(\%)$ & PA $(\%)$ & DA $(\%)$ \\
\hline Padrão & $5,75 \pm 0,22$ & $12,76 \pm 0,48$ & $2,22 \pm 0,03$ \\
C1 & $3,97 \pm 0,12$ & $8,87 \pm 0,26$ & $2,23 \pm 0,01$ \\
C2 & NA & NA & NA \\
C3 & $4,58 \pm 0,11$ & $10,19 \pm 0,28$ & $2,22 \pm 0,01$ \\
C4 & $3,79 \pm 0,37$ & $8,14 \pm 0,74$ & $2,15 \pm 0,02$ \\
\hline
\end{tabular}

NA - não avaliado.
Tabela V - Resultados dos ensaios físicos com $28 \mathrm{~d}$. [Table V-Results for 28 days physical essays.]

\begin{tabular}{cccc}
\hline Material & AA $(\%)$ & PA $(\%)$ & DA $(\%)$ \\
\hline Padrão & $5,58 \pm 0,25$ & $12,41 \pm 0,58$ & $2,22 \pm 0,02$ \\
C1 & $3,92 \pm 0,26$ & $8,75 \pm 0,56$ & $2,23 \pm 0,01$ \\
C3 & $6,42 \pm 1,13$ & $14,09 \pm 2,09$ & $2,21 \pm 0,09$ \\
C4 & $5,73 \pm 0,25$ & $12,09 \pm 0,41$ & $2,11 \pm 0,02$ \\
\hline
\end{tabular}

foram avaliadas a absorção de água, porosidade aparente e densidade aparente para essa composição. A Tabela V apresenta os resultados dos ensaios físicos de absorção de água, porosidade aparente e densidade aparente dos corpos de prova do padrão, do proposto 1 , proposto 3 e proposto 4 com tempo de cura de $28 \mathrm{~d}$.

As composições propostas C1, C3 e C4 apresentaram menores absorção de água e porosidade aparente do que o padrão para $7 \mathrm{~d}$. Com $28 \mathrm{~d}$; apenas a composição $\mathrm{C} 1$ apresentou valores menores, enquanto que as composições C3 e C4 apresentaram valores próximos dos da composição padrão. Em termos de densidade aparente, a composição C4 apresentou menor densidade aparente que as demais composições ( $\mathrm{C} 1, \mathrm{C} 3$ e P), tanto para $7 \mathrm{~d}$ quanto para $28 \mathrm{~d}$. As composições $\mathrm{C} 1$ e C3 apresentaram valores de densidade aparente próximos à composição padrão.

A Fig. 1 mostra as tendências das composições propostas e do padrão em relação ao ensaio físico de absorção de água com 7 e $28 \mathrm{~d}$.

\section{ENSAIO FÍSICO DE ABSORÇÃO DE ÁGUA}

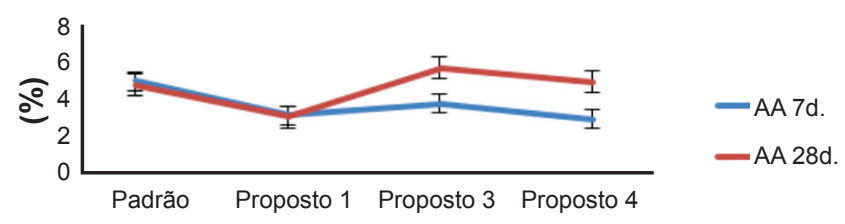

Figura 1: Resultados de ensaio físico de absorção de água (AA). [Figure 1: Water absorption results (WA).]

A Fig. 2 mostra as tendências das composições propostas e do padrão em relação ao ensaio físico de porosidade aparente com 7 e $28 \mathrm{~d}$.

A Fig. 3 mostra as tendências das composições propostas

\section{ENSAIO FÍSICO DE POROSIDADE APARENTE}

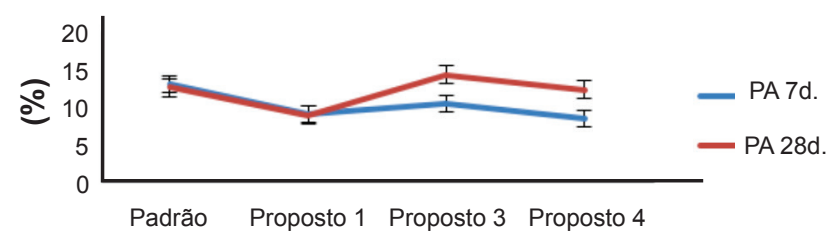

Figura 2: Resultados de ensaio físico de porosidade aparente (PA). [Figure 2: Apparent porosity results (AP).] 


\section{ENSAIO FÍSICO DE DENSIDADE APARENTE}

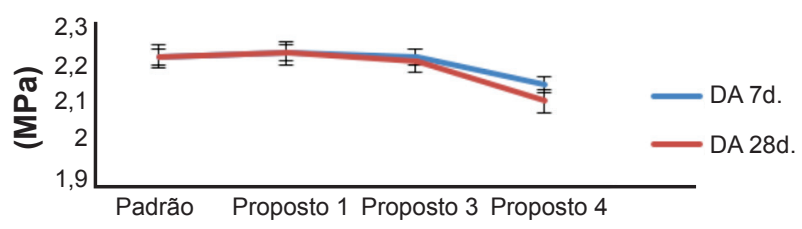

Figura 3: Resultados de ensaio físico de densidade aparente (DA). [Figure 3: Apparent gravity results $(A G)$.]

Tabela VI - Resultados do ensaio mecânico de resistência à compressão $\left(\mathrm{f}_{\mathrm{pk}}\right)$.

[Table VI - Compression strength $\left(f_{p k}\right)$ results.]

\begin{tabular}{ccc}
\hline $\begin{array}{c}\text { Corpo de } \\
\text { Prova }\end{array}$ & $\begin{array}{c}\text { Limite de } \\
\text { resistência à } \\
\text { compressão }(\mathrm{MPa})\end{array}$ & $\begin{array}{c}\text { Tipo de } \\
\text { ruptura }\end{array}$ \\
\hline Padrão & $20,59 \pm 2,90$ & Cônica e Bipartida \\
C1 & $19,49 \pm 2,09$ & Cônica e Bipartida \\
C3 & $27,39 \pm 2,77$ & Cônica e Bipartida \\
C4 & $20,45 \pm 3,53$ & Cônica e Bipartida \\
\hline
\end{tabular}

e do padrão em relação ao ensaio físico de densidade aparente com $7 \mathrm{~d}$ e $28 \mathrm{~d}$.

Comparando a composição $\mathrm{C} 1$ ao padrão com tempo de cura de $7 \mathrm{~d}$ em temperatura ambiente, esse apresentou uma redução de $1,78 \%$ na absorção de água, $3,89 \%$ na porosidade aparente e $0,01 \%$ na densidade aparente, enquanto que com tempo de cura de $28 \mathrm{~d}$ em temperatura ambiente, esse apresentou uma redução de $1,66 \%$ na absorção de água, $3,66 \%$ na porosidade aparente e $0,01 \%$ na densidade aparente. Uma vez que houve significativa redução na absorção de água e na porosidade aparente para essa composição, o concreto alternativo poderia, por exemplo, ser utilizado para a moldagem de baldrames em alicerces de construções, objetivando reduzir futuros problemas com umidade nas paredes.

A Tabela VI apresenta os resultados dos ensaios mecânicos, dos corpos de prova das composições P, C1, C3 e $\mathrm{C} 4$ com tempo de cura de $28 \mathrm{~d}$. Não foram realizados ensaios de compressão para os corpos de prova com sete dias de cura

\section{ENSAIO MECÂNICO DE RESISTÊNCIA À COMPRESSÃO}

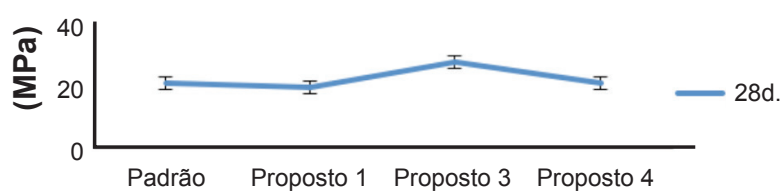

Figura 4: Resultados de limite de resistência à compressão $\left(\mathrm{f}_{\mathrm{pk}}\right)$. [Figure 4: Compression strength graph $\left(f_{p k}\right)$.]

por não ser interessante avaliar essa propriedade com esse tempo de cura para produtos acabados com possibilidade de comercialização.

A composição C3 apresentou valores superiores de limite de resistência à compressão às demais composições (P, C1 e C4), enquanto que as composições C1 e C4 apresentaram valores próximos aos valores da composição padrão. Com relação ao tipo de ruptura, todos os corpos de prova das composições apresentaram ruptura do tipo cônica e bipartida.

A Fig. 4 ilustra a tendência das composições propostas e do padrão em relação ao limite de resistência à compressão (LRC).

Os concretos são classificados em grupos de resistência, grupo I e grupo II, conforme o limite de resistência à compressão (LRC) segundo a NBR 8953 [1].Com base nas especificações da NBR 8953 [1], a composição C1 apresentou resultados mecânicos satisfatórios para ser utilizado na construção civil, porque mesmo sem a adição de brita em sua composição demonstrou propriedades semelhantes ao concreto C20 grupo I de resistência, que possui brita em sua composição. A brita é considerada um material inerte e aumenta a resistência à compressão no concreto endurecido. O concreto $\mathrm{C} 20$ é utilizado atualmente na construção civil para moldagem de pilares, vigas, lajes e fundações. Desta forma a composição do proposto 1 com adição de brita pode ser destinada para as mesmas finalidades.

Custo por metro cúbico de concreto: como a amostra $\mathrm{C} 1$ foi a que obteve melhores resultados de absorção de água e porosidade aparente, além de resultados próximos da composição padrão para densidade aparente e para o limite de resistência à compressão, essa foi utilizada para realizar

Tabela VII - Dosagem e custo do concreto comercial.

[Table VII - Commercial concrete dosage and expense.]

\begin{tabular}{|c|c|c|c|c|c|}
\hline \multicolumn{6}{|c|}{ Dosagem para $1 \mathrm{~m}^{3}$ de concreto com cimento CP II E - 32} \\
\hline Tempo de & & & Quantidade de Materiais & & \\
\hline $\begin{array}{c}\text { cura em } \\
\text { dias }\end{array}$ & Cimento $(\mathrm{kg})$ & Areia (L) & Agregado graúdo (L) & Água (L) & Aditivo (L) \\
\hline 20 & 269 & 629 & 560 & 196 & 4 \\
\hline Custo por produto $\mathrm{R} \$$ & 105,00 & 48,00 & 42,00 & 0,20 & 5,00 \\
\hline & & Custo & $\mathrm{R} \$ 200,20$ & & \\
\hline
\end{tabular}


Tabela VIII- Dosagem e custo do concreto alternativo. [Table VIII - Alternative concrete dosage and expense.]

\begin{tabular}{|c|c|c|c|c|c|c|c|c|}
\hline \multicolumn{9}{|c|}{ Dosagem para $1 \mathrm{~m}^{3}$ de concreto alternativo } \\
\hline \multirow{2}{*}{$\begin{array}{l}\text { Tempo de } \\
\text { cura em dias }\end{array}$} & \multicolumn{8}{|c|}{ Quantidade de Materiais } \\
\hline & $\begin{array}{l}\text { Cimento } \\
(\mathrm{kg})\end{array}$ & $\begin{array}{l}\text { Escória } \\
(\mathrm{kg})\end{array}$ & $\begin{array}{l}\text { Areia } \\
\text { (L) }\end{array}$ & $\begin{array}{c}\text { Areia de } \\
\text { Macharia (L) }\end{array}$ & Pedra (L) & Água (L) & $\begin{array}{l}\text { Aditivo } \\
\text { (L) }\end{array}$ & $\begin{array}{c}\text { Resina (PVC) } \\
(\mathrm{kg})\end{array}$ \\
\hline 28 & 258 & 11 & 314,5 & 314,5 & 560 & 196 & 6,5 & 82 \\
\hline $\begin{array}{c}\text { Custo por produto } \\
\mathrm{R} \$\end{array}$ & 100,00 & 0,00 & 24,00 & 0,00 & 42,00 & 0,20 & 8,00 & 492,00 \\
\hline
\end{tabular}

a comparação do custo comercial de fabricação de $1 \mathrm{~m}^{3}$ de concreto com relação à composição padrão. A Tabela VII mostra a dosagem de materiais e o custo de $1 \mathrm{~m}^{3}$ de concreto atualmente comercializado.

A Tabela VIII mostra a dosagem de materiais e o custo de $1 \mathrm{~m}^{3}$ de concreto alternativo.

A resina de Poli (cloreto de vinila) (PVC) NORVIC P72HA, aumentou de forma considerável o custo do concreto alternativo, onerando o custo em $\mathrm{R} \$ 466,00$ por metro cúbico com relação ao concreto padrão, não sendo, dessa forma, viável a comercialização da composição proposta $\mathrm{C} 1$.

\section{CONCLUSÕES}

As composições propostas para concreto alternativo apresentaram propriedades segundo as especificações da NBR 8953 [1], para ser utilizado na construção civil, mas, sua produção ainda não é atrativa devido ao preço da resina de Poli (cloreto de vinila) (PVC) NORVIC P72HA adicionada á composição proposta. A composição que apresentou melhores resultados de propriedades físico-mecânicas foi a composição $\mathrm{C} 1$. Os resultados da incorporação da areia de macharia e escória de forno a indução foram bem sucedidos e satisfatórios, e dessa forma pode-se reduzir o seu descarte em aterros sanitários e promover o desenvolvimento sustentável, para satisfazer as necessidades da geração atual, sem comprometer as gerações futuras.

\section{AGRADECIMENTOS}

Ao Laboratório de Biomecânica (Profa. Dra. C.A.C. Zavaglia) e ao Laboratório de Metalurgia Física e Solidificação (Prof. Dr. R. Caram Jr.) da Faculdade de Engenharia Mecânica da UNICAMP.

\section{REFERÊNCIAS}

[1] Associação Brasileira de Normas Técnicas, "Concreto para fins estruturais - Classificação pela massa específica, por grupos de resistência e consistência”, NBR 8953 (2009). [2] Presidência da República, Casa Civil, Subchefia para Assuntos Jurídicos, Lei 12305(02/08/2010),disponível emhttp://www.planalto.gov.br/ccivil_03/_ato20072010/2010/lei/112305.htm. Acesso em 09/01/2015.

[3] A.B. Fagundes, C.R. Vaz, I.L. de Oliveira, J.L. Kovaleski,
Gepros: Gestão da produção, operações e sistemas 5, 2 (2010) 27-40.

[4] R.L.P. Carnin, C.L. Silva, R.J. Pozzi, D. Cardoso Jr., M. V. Folgueras, W. Malkowski, Pavimentação 19 (2010) 6071.

[5] W.V. Srubar III, Cem. Concr. Composites 55 (2015) 103111.

[6] Associação Brasileira de Normas Técnicas, "Resíduos sólidos- classificação", NBR 10004 (2004).

[7] Companhia de Tecnologia de Saneamento Ambiental - CETESB, Decisão de Diretoria No 152/2007/C/E, de 08/09/2007 disponível em http://www.cetesb.sp.gov.br/ media/s/2010/06/21/20100621111349_14c906d046b6f3c90 4a8e859375107c5.pdf. Acesso em 09/01/2015.

[8] C.E.Valle, “Qualidade Ambiental; ISO 14.000”, S. Paulo, SENAC (2002).

[9] R.L. Pereira, C.J. Cunha, F.N.P. Mattoso, L. Momm, "Reaproveitamento de resíduo de areia verde de fundição como agregado em misturas asfálticas", Anais $12^{\circ}$ Cong. Fundição, S. Paulo, SP (2005).

[10] S.M. Biolo, "Reuso do resíduo de fundição areia verde na produção de blocos cerâmicos", Diss. Mestrado, Escola de Engenharia, Universidade Federal do Rio Grande do Sul (2005) $162 \mathrm{f..}$

[11] L.M.G. Klinsky, "Proposta de reaproveitamento de areia de fundição em sub-bases e bases de pavimentos flexíveis, através de sua incorporação a solos argilosos", Diss. Mestrado. Escola de Engenharia de S. Carlos, Universidade de S. Paulo (2008) $215 \mathrm{f}$.

[12] K.B. Guerino, J. Vicenzi, S.R. Bragança, C.P. Bergmann, "Uso de areia de fundição como matéria prima para a produção de cerâmicas brancas triaxiais", Ceram. Ind. 15 (2010) 42-46.

[13] D.F. Oliveira, "Caracterização dos finos de areia de uma fundição e sua incorporação em argamassa de cimento", Diss. Mestrado, Faculdade de Engenharia de Bauru, Universidade Estadual Paulista (2010) $70 \mathrm{f}$.

[14] L.C. Armange, L.F. Neppel, E. Gemelli, N.H.A. Camargo, "Utilização de areia de fundição residual para uso em argamassa", Rev. Matéria 10, 1 (2005) 51-62.

[15] R.L.C. Carnin, C.L. Silva, R.J. Pozzi, J.R.D. Cardoso, M. V. Folgueras, W. Malkowski, "Desenvolvimento de peças de concreto (paver) contendo areia descartada de fundição para pavimento intertravado", Rev. Pavimentação, ano V (2010) 56-67. 
[16] G. Penkaitis, J.B. Sígolo, "Waste fround sand. Environmental implication and characterization",Geologia USP, Série científica 12, 3 (2012) 57-70.

[17] Associação Brasileira de Normas Técnicas, "Concreto - Procedimento para moldagem e cura de corpos de prova", NBR 5738(2003).
[18] Associação Brasileira de Normas Técnicas, "Concreto - Ensaios de compressão de corpos de prova cilíndricos", NBR 5739: 2007.

[19] P. Souza Santos, Ciência e tecnologia de argilas, Vol. 1, Edgard Blucher Ltda., S. Paulo, SP (1989).

(Rec. 28/06/2015, Rev. 15/07/2015, Ac. 15/07/2015) 